Publ. RIMS, Kyoto Univ.

21 (1985), 719-735

\title{
A Measure of Integrity for Local Analytic Algebras
}

By

\author{
Shuzo IZUMI*
}

\section{§ 0. Introduction}

We will complete the study $\left[\mathrm{I}_{1}\right],\left[\mathrm{I}_{2}\right]$ on orders of elements of a local analytic algebra. Let $\left(X, \mathcal{O}_{X}\right)$ be a complex space and $(\mathcal{O}, \mathfrak{m})$ $:=\left(\mathcal{O}_{X, \xi}, \mathfrak{m}_{\xi}\right)$ its local algebra at $\xi \in X$. $\mathcal{O}$ can be expressed as $\mathcal{O}=$ $\boldsymbol{C}\{x\} / I$ for some ideal $I$ of the algebra $\boldsymbol{C}\{x\}$ of convergent power series in $x=\left(x_{1}, \cdots, x_{m}\right)$. We define three kinds of orders for $f \in \mathcal{O}$.

algebraic order: $\nu(f)=\nu_{\xi}(f):=\sup \left\{p: f \in \mathfrak{m}^{p}\right\}=\sup \{$ the degree of the lowest non-zero homogeneous term of $\hat{f}: \hat{f}$ is a representative of $f$ in $C\{x\}\}$

reduced order $\left(\left[\mathrm{Re}_{1}\right]\right.$, cf. $\left.[\mathrm{S}]\right): \bar{\nu}(f):=\lim _{k \rightarrow \infty} \nu\left(f^{k}\right) / k$

analytic order along $A \subset|X|: \mu_{A, \xi}(f):=\sup \{p$ : there exist $\alpha>0$, neighbourhood $U$ of $\xi$ and representative $\tilde{f}$ of $f$ over $U$ such that $|\tilde{f}(x)|$ $\left.\leqq \alpha|x-\xi|^{p} \quad(x \in U \cap A)\right\}$.

We can easily verify the following. inequality for the reduced order:

$$
\left(\mathrm{I}_{0}\right) \quad \bar{\nu}(f) \geqq \nu(f) \quad(f \in \mathcal{O}) .
$$

inequality for products:

$$
\left(\mathrm{I}_{1}\right) \quad \nu(f g) \geqq \nu(f)+\nu(g) \quad(f, g \in \mathcal{O}) .
$$

inequality for pullbacks: If $\Phi: Y \rightarrow X$ is a morphism such that $\Phi(\eta)=\xi$,

$$
\left(\mathrm{I}_{2}\right) \quad \nu_{\eta}(f \circ \Phi) \geqq \nu_{\xi}(f) \quad(f \in \mathcal{O}) .
$$

inequality for the analytic order:

$$
\left(\mathrm{I}_{3}\right) \quad \mu_{A, \xi}(f) \geqq \nu(f) \quad(f \in \mathcal{O}) .
$$

We are interested in linear complementary inequalities (LCI) of

Communicated by H. Hironaka, June 20, 1984. Reviced October 9, 1984.

* Faculty of Science and Technology, Kinki-University, Higashi-Osaka 577, Japan. 
these $\left(I_{i}\right)$. By the strong valuation theorem of $\operatorname{Rees}\left[\mathrm{Re}_{3}\right]$ on Noetherian rings, we see the following:

If $X$ is reduced at $\xi$, there exists $b_{0}=b_{0}(\mathcal{O}) \geqq 0$ such that

$$
\left(\mathrm{GI}_{0}\right) \quad \bar{\nu}(f) \leqq \nu(f)+b_{0} \quad(f \in \mathcal{O}) .
$$

We prove the following LGI as our main theorem, (3.4), assuming that $X$ is reduced and irreducible at $\xi$ i. e. $\mathcal{O}$ is an integral domain. (1) There exist $a_{1}=a_{1}(\mathcal{O}) \geqq 1, b_{1}=b_{1}(\mathcal{O}) \geqq 0$ such that

$$
\left(\mathrm{CI}_{1}\right) \quad \nu(f g) \leqq a_{1}(\nu(f)+\nu(g))+b_{1} \quad(f, g \in \mathcal{O}) .
$$

(2) For any morphism $\Phi:(Y, \eta) \rightarrow(X, \xi)$ with $\operatorname{dim} X_{\xi}=\operatorname{grnk}_{\eta} \Phi$ (see below), there exist $a_{2}=a_{2}(\Phi) \geqq 1, b_{2}=b_{2}(\Phi) \geqq 0$ such that

$$
\left(\mathrm{CI}_{2}\right) \quad \nu_{\eta}(f \circ \Phi) \leqq a_{2} \nu_{\xi}(f)+b_{2} \quad(f \in \mathcal{O}) .
$$

(3) For any open subanalytic set $A \subset|X|$ adherent to $\xi$, there exist $a_{3}=$ $a_{3}(A) \geqq 1, b_{3}=b_{3}(A) \geqq 0$ such that

$$
\left(\mathrm{CI}_{3}\right) \quad \mu_{A, \xi}(f) \leqq a_{3} \nu(f)+b_{3} \quad(f \in \mathcal{O}) .
$$

In the above $\operatorname{grnk}_{\eta} \Phi$ denotes the generic rank defined for a morphism of germs of analytic spaces over $k=C$ or $\mathbb{R}$ :

$\operatorname{grnk}_{\eta} \Phi=\varepsilon \cdot \inf \{$ topological dimension of $\Phi(U): U$ is a neighbourhood of $\eta\} \quad(\varepsilon=1 / 2$ if $k=C ; \varepsilon=1$ if $k=\mathbb{R})$.

This has been used to study properties of a ring homomorphism induced by a morphism between spaces (see [Be]).

The constants $a_{i}$ (or $b_{i}$ ) in the above LCI measure some intrinsic properties of $\mathcal{O}, \Phi$ and $A$ as follows. If $\mathcal{O}$ is not an integral domain, $a_{1}$ does not exist $\left(a_{1}=\infty\right)$. So we may consider $\inf a_{1}=\inf \left\{a_{1}\right.$ : there exists $b_{1}$ such that $\left(\mathrm{CI}_{1}\right)$ holds $\}$ as a measure of integrity of the algebra $\mathcal{O}$ (or $1 /$ inf $a_{1}$ as a distance of $\mathcal{O}$ from non-integral domains). inf $a_{2}$ can be considered as a kind of order of the morphism $\Phi$. Indeed, in case $(X, \xi)=(C, 0)$, we see that inf $a_{2}$ coincides with the reduced order, $\bar{\nu}(\Phi)$, of the function $\Phi$. inf $a_{3}$ seems to have a relation with the size of the germ $A_{\xi}$. Of course, if $A \subset B$, inf $a_{3}(A) \geqq \inf a_{3}(B)$.

We have already proved that (1) and (2) of the main theorem are equivalent $\left(\left[\mathrm{I}_{1}\right],(1.2)\right)$. So we prove (1) by induction on $n:=$ $\operatorname{dim} X_{\xi}$. (The proof of (3) is postponed till the end of $\S 4$.) In $\S 1$ we recall the valuation theorem in a special form we need later. It implies the case $n=1$ of (1) immediately. The case $n=2$ can not be reduced to the case $n=1$. We can manage to prove this case by 
the use of the intersection matrix of Du Val-Mumford for a normal surface $(\S 2)$. In $\S 3$ we reduce the case $n(\geqq 3)$ to the case $n-1$ using a Bertini type theorem of Flenner, completing the proof of (1). In $\S 4$ we show a real analytic version of the main theorem. There we remark that Risler's criterion for real ideal (=real radical) gives an affirmative answer to Malgrange's conjecture ([Ma], p. 124) on the real analytic set associated to a complex analytic set. In $\S 5$, as an application of the main theorem, we treat "near solutions" of the algebraic equation $f^{p} u-g^{p}=0$ in $\mathbb{C}[[x]]$ for a given $u \in \mathbb{C}\{x\}$ and a prime number $\mathrm{p}$. This yields an intrinsic measure of nearness of $u$ to $p$-th powers in $\mathbb{C}\{x\}$ In Appendix we calculate the explicit values of inf $a_{1}$ and inf $b_{1}$ for some singularities.

The author wishes to thank Mr. M. Tomari for many helpful advices and Professor K. Saito for pointing out a mistake in the formulation of (4.5) in the provisional manuscript.

\section{$\S 1$. The Valuation Theorem}

Here we introduce the most basic result on orders on an analytic algebra, restricting ourselves to the case of the orders with respect to the maximal ideal. Let $X$ be a positive dimensional complex space reduced at $\xi$ and let $\mathscr{M}$ denote the class of all morphisms $\Pi: Y \rightarrow X$ such that $Y$ are normal, $I I$ induce proper morphisms onto neighbourhoods of $\xi$ and the ideal sheaves defining $\Pi^{-1}(\xi)$ (with the canonical structure) are invertible. $\mathscr{M}$ is not empty; the composition of the normalization after the blowing-up with center $\xi$ belongs to $\mathscr{M}$. If $\Pi \in \mathscr{M}$, the exceptional fiber $E$ over $\xi\left(=\right.$ the reduction of $\Pi^{-1}(\xi)$ ) has a finite number of irreducible components $\left\{E_{i}\right\}_{i \in A(I)}$. Let $\mathscr{I}$ and $\mathscr{I}_{i}$ denote the sheaves of ideals defining $E$ and $E_{i}$ respectively. By the normality and the Hauptidealsatz (cf. [G-R], p. 129, p. 223), there exists an complex analytic set $S \subset|Y|$ of codimension at least 2 such that $Y-S\left(=\left.Y\right|_{|Y|-S}\right)$ is a smooth manifold and $E-S$ and $E_{i}-S$ are submanifolds of $Y-S$ of codimension 1. For $f \in \Gamma\left(U, \mathcal{O}_{X}\right)$, we put

$$
v_{i}(f):=\sup \left\{p: \quad(f \circ \Pi)_{y} \in \mathscr{I}_{i}^{p} \text { for all (some) } y \in\left|E_{i}\right|-S\right\} .
$$

If $f \in \mathcal{O}:=\mathcal{O}_{X, \xi}$, we define $v_{i}(f)$ using its representative. It is obvious that $v_{i}(f g)=v_{i}(f)+v_{i}(g) \quad(f, g \in \mathcal{O})$. 
(1.1) Lemma. ([L-T],(4.1.8)). If we put $v_{i}(\mathfrak{m})=\inf \left\{v_{i}(g): g \in \mathfrak{m}\right\}$ $\left(\mathfrak{m}:=m_{\xi}\right)$, we have

$\inf \left\{v_{i}(f) / v_{i}(\mathfrak{m}): i \in A(\Pi)\right\}=\sup \left\{p / q:(f \circ \Pi)^{q} \in \Gamma\left(U, \mathscr{I}^{p}\right)\right\}$.

(1.2) Lemma. ([L-T], (4. 1.6), (5.5), (7.2) cf. (1.12)). Let X be a positive dimensional complex space reduced at $\xi$. Then, for any $f \in$ $\Gamma\left(X, \mathcal{O}_{X}\right), \bar{\nu}(f)$ is a rational number and the following conditions are equivalent for any $p, q \in \mathbb{N}$.

(r) $\quad \bar{\nu}(f) \geqq p / q$.

(i) There exist $\sigma_{i} \in \mathcal{O}(1 \leqq i \leqq k)$ such that $\nu\left(\sigma_{i}\right) \geqq i p / q$ and

$$
f^{k}-\sigma_{1} f^{k-1}+\sigma_{2} f^{k-2}-\cdots \pm \sigma_{k}=0 \text { in } \mathcal{O} \text {. }
$$

(c) Let $D$ denote the unit open disc in the complex plane $C$. Then for any morphism $\Phi: D \rightarrow X$ with $\Phi(0)=\xi$, we have

$$
\nu_{0}(f \circ \Phi) \geqq(p / q) \inf \left\{\nu_{0}(g \circ \Phi): g \in \mathfrak{m}\right\} .
$$

(m) For any $\Pi: Y \rightarrow X$ in $\mathscr{M}$,

$$
\inf \left\{v_{i}(f) / v_{i}(\mathfrak{m}): i \in A(\Pi)\right\} \geqq p / q .
$$

(m') There exists $\Pi: Y \rightarrow X$ in $\mathscr{M}$ such that

$$
\inf \left\{v_{i}(f) / v_{i}(\mathfrak{M}): i \in A(\Pi)\right\} \geqq p / q .
$$

(a) $\mu_{|X|, \xi}(f) \geqq p / q$.

(1.3) Remark. This theorem was proved in an algebraic form by Rees $\left[\mathrm{Re}_{1}\right],\left[\mathrm{Re}_{2}\right]$ and Nagata $\left[\mathrm{N}_{1}\right]$ independently, developing Samuel's paper [S]. They treated more general rings. The conditions (c) and (a) were considered first by Lejeune and Teissier. They stated the result for general coherent sheaves on complex spaces.

(1.4) Remark. By $(m)$ and $\left(m^{\prime}\right)$ we see that $\bar{\nu}(f)=\inf \left\{v_{i}(f) / v_{i}(\mathfrak{m})\right.$ : $i \in A(\Pi)\}$ for any $\Pi \in \mathscr{M}$.

\section{\$ 2. LCI for Normal Surfaces}

Here we use the notation in $\S 1$.

(2.1) Theorem. If $X$ is a 2-dimensional complex space normal at $\xi$, $\Pi: Y \rightarrow X$ a morphism in $\mathscr{M}$ and if $Y$ is nonsingular, we have the following.

(i) There exist $c_{i j}>0\left(c_{i i}=1\right)$ such that $c_{i j} v_{j}(f) \geqq v_{i}(f)(i, j \in A(\Pi))$. 
(ii) If we put $a_{1}=\left(1+\max c_{i j} v_{j}(\mathfrak{m}) / v_{i}(\mathfrak{m})\right) / 2$, we have $\bar{\nu}(f g) \leqq$ $a_{1}(\bar{\nu}(f)+\bar{\nu}(g))$ and hence $\nu(f g) \leqq a_{1}(\nu(f)+\nu(g))+2 a_{1} b_{0}(f, g \in \mathcal{O})$ by $\left(\mathrm{CI}_{0}\right)$.

Proof. (i) Let $A(\Pi)=\left\{E_{1}, \cdots, E_{k}\right\}$ and let $S_{f}$ denote the strict transform of the divisor $(f)$ of $f \in \mathcal{O}$ with respect to $\Pi$. Then no $E_{i}$ appears in $S_{f}$ and $(f \circ \Pi)=S_{f}+\sum m_{i} E_{i}\left(m_{i}=v_{\imath}(f)\right)$. Taking intersection number with $E_{j}$ around $E$ (cf. $\left.[\mathrm{Mu}]\right)$, we have

$$
\sum_{i} m_{i} e_{i j}=(f \circ \Pi) \cdot E_{j}-S_{f} \cdot E_{j}=-S_{f} \cdot E_{j} \leqq 0 \quad\left(j=1, \cdots, k ; e_{i j}=E_{i} \cdot E_{j}\right) .
$$

Hence the vector $\left(\mathrm{m}_{1}, \cdots, \mathrm{m}_{k}\right)$ is contained in the convex cone $K \subset \mathbb{R}^{k}$ defined by

$$
\sum_{i} x_{i} e_{i j} \leqq 0, \quad x_{j} \geqq 0 \quad(j=1, \cdots, k) .
$$

We have only to prove that $K \backslash\{0\}$ is included in the strictly positive quadrant. Assume the contrary. By a rearrangement of the coordinates, we may assume that $\left(x_{1}, \cdots, x_{p}, 0, \cdots, 0\right) \in K\left(x_{1}, \cdots, x_{p}>0,1 \leqq p<k\right)$. Since $e_{i j} \geqq 0 \quad(i \neq j),(*)$ implies $e_{i j}=0 \quad(i \leqq p, j>p)$. This contradicts to the fact that $E$ is connected.

(ii) We may assume that $v_{\alpha}(f) / v_{\alpha}(\mathfrak{m})=\bar{\nu}(f) \leqq \bar{\nu}(g)=v_{\beta}(g) / v_{\beta}(\mathfrak{m})$ (see (1.4)). Then we have

$$
\begin{aligned}
\bar{\nu}(f g) & =\min \left(v_{i}(f)+v_{i}(g)\right) / v_{i}(\mathfrak{M}) \leqq\left(v_{\beta}(f)+v_{\beta}(g)\right) / v_{\beta}(\mathfrak{M}) \\
& \leqq c_{\beta \alpha}\left(v_{\alpha}(\mathfrak{M}) / v_{\beta}(\mathfrak{M})\right) v_{\alpha}(f) / v_{\alpha}(\mathfrak{M})+v_{\beta}(g) / v_{\beta}(\mathfrak{M}) \\
& \leqq\left(2 a_{1}-1\right) \bar{\nu}(f)+\bar{\nu}(g) \leqq a_{1}(\bar{\nu}(f)+\bar{\nu}(g))
\end{aligned}
$$

(2. 2) Remark. $c_{i j}$ are obviously determined by $\left(e_{i j}\right)$. We can show that $a_{1}$ is also majorized by a constant determined by $\left(e_{i j}\right)$. Indeed there exists $f \in \mathcal{O}_{X, \xi}$ such that $v_{i}(f)=v_{i}(\mathfrak{m})(i=1, \cdots, k)$. Then $v_{j}(\mathfrak{m}) \leqq$ $c_{j i} v_{i}(\mathfrak{m})$ and $a_{1} \leqq\left(1+\max _{i, j} c_{i j} c_{j i}\right) / 2$.

\section{§3. LCI in General Case}

We borrow an important result, a Bertini type theorem of Flenner:

(3.1) Lemma $([\mathrm{F}],(4.10))$. Let $(\mathcal{O}, \mathfrak{m})$ be a local $k$-algebra over a field $k$ of characteristic 0 and let $\varphi_{1}, \cdots, \varphi_{m} \in m$. Suppose that the completion $\hat{\mathcal{O}}$ is an integral domain and $\operatorname{dim} \mathcal{O}_{p} \geqq 3$, Prof $\mathcal{O}_{p} \geqq 2$ for any prime ideal $p$ that contains $\varphi_{1}, \cdots, \varphi_{m}\left(\mathcal{O}_{p}:\right.$ the localization $)$. Then for any $e=$ 
$\left(e_{1}, \cdots, e_{m}\right)$ in a nonempty Zariski open subset of $k^{m}, \sum e_{i} \varphi_{i}$ is a prime element of $\mathcal{O}($ even of $\hat{\mathcal{O}})$.

(3.2) Lemma. Let $B$ be an infinite set of complex hyperplanes (linear subspaces of codimension 1) through 0 in $\mathbb{C}^{m}$. Then $\nu_{0}(f)=\min _{H \in B} \nu_{0}(f \mid H)$ $(f \in \boldsymbol{C}\{x\})$.

Proof. The inequality $\nu_{0}(f) \leqq \nu_{0}(f \mid H)$ is a special case of the inequality for pullbacks $\left(I_{2}\right)$. The case $f=0$ is trivial. If $f \neq 0$ and if $H$ is not contained in the tangent cone of the hypersurface defined by $f$, then the initial form (the non-zero homogeneous term of the lowest degree) survives after restriction to $H$ and $\nu_{0}(f)=\nu_{0}(f \mid H)$. Since $B$ is an infinite set, such an $H$ really exists in $B$.

(3.3) Lemma. ( $\left[\mathrm{I}_{1}\right]$, Added in proof). Let $\Phi:(Y, \eta) \rightarrow(X, \xi)$ be the normalization of a reduced and irreducible germ of a complex space. If $(Y, \eta)$ satisfies $\left(\mathrm{CI}_{1}\right)$ in $(3.4),(X, \xi)$ also satisfies it.

Proof. Since $\Phi$ is finite $\left(\mathrm{CI}_{2}\right)$ holds $\left(\left[\mathrm{I}_{1}\right],(2.3)\right.$ or $\left.(4.1)\right): \nu_{n}(f \circ \Phi)$ $\leqq a \nu_{\xi}(f)+b \quad\left(f \in \mathcal{O}_{X, \xi}\right)$ for some $a \geqq 1$ and $\mathrm{b} \geqq 0$. Then we have

$$
\begin{aligned}
\nu_{\xi}(f g) & \leqq \nu_{\eta}((f \circ \Phi)(g \circ \Phi)) \leqq a_{1}\left(\nu_{\eta}(f \circ \Phi)+\nu_{\eta}(g \circ \Phi)\right)+b_{1} \\
& \leqq a_{1} a\left(\nu_{\xi}(f)+\nu_{\xi}(g)\right)+2 a_{1} b+b_{1} \quad\left(f, g \in \mathcal{O}_{X, \xi}\right) .
\end{aligned}
$$

(3.4) Main Theorem. Let $X$ be a complex space reduced and irreducible at $\xi$ (i.e. $\mathcal{O}:=\mathcal{O}_{X, \xi}$ is an integral domain) such that $n:=\operatorname{dim} X_{\xi} \geqq 1$. Then it has the following three properties.

(1) There exist $a_{1}=a_{1}(\mathcal{O}) \geqq 1, b_{1}=b_{1}(\mathcal{O}) \geqq 0$ such that

$\left(\mathrm{CI}_{1}\right) \quad \nu(f g) \leqq a_{1}(\nu(f)+\nu(g))+b_{1} \quad(f, g \in \mathcal{O})$.

(2) For any morphism $\Phi:(Y, \eta) \rightarrow(X, \xi)$ with $\operatorname{grnk}_{\eta} \Phi=\operatorname{dim} X_{\xi}$ (see $\S 0$ for the symbol grnk), there exist $a_{1}=a_{1}(\mathcal{O}) \geqq 1, b_{1}=b_{1}(\mathcal{O}) \geqq 0$ such that

$\left(\mathrm{CI}_{2}\right) \quad \nu_{\eta}(f \circ \Phi) \leqq a_{2} \nu_{\xi}(f)+b_{2} \quad(f \in \mathcal{O})$.

(3) For any open subanalytic set $A \subset X$ adherent to $\xi$, there exist $a_{3}=$ $a_{3}(A) \geqq 1, b_{3}=b_{3}(A) \geqq 0$ such that

$$
\left(\mathrm{CI}_{3}\right) \quad \mu_{A, \xi}(f) \leqq a_{3} \nu(f)+b_{3} \quad(f \in \mathcal{O}) .
$$


Proof. The proof of (3) is postponed to (4. 8). Since (1) and (2) are equivalent $\left(\left[\mathrm{I}_{1}\right],(1.2)\right)$, we have only to prove one of them. The case $n=1$ of (1) follows from (1.4), since $v_{i}(f g)=v_{i}(f)+v_{i}(g)$ and $\# A(\pi)=1$. The case $n=2$ follows from (2.1) and (3.3). As to the case $n \geqq 3$, we. prove (1) by induction on $n$.

Suppose that the case $n-1 \quad(n \geqq 3)$ has been proved already. We may assume that $X$ is normal by (3.3). We may also assume that $\xi=0 \in \mathbb{C}^{m}$ and $X$ is a closed complex subspace of a product open neighbourhood $U=V \times W\left(V \subset \mathbb{C}^{n}, W \subset \mathbb{C}^{m-n}\right)$ of 0 such that the projection $\Pi: X \rightarrow V$ is finite proper open and locally biholomorphic on $X-\Pi^{-1}(D)$ for some proper analytic subset $D \subset V$ (local parametrization theorem). If we choose a sufficiently small $U$ and such a coordinate space $\boldsymbol{C}^{m-n}$ that intersects the tangent cone of $X_{0}$ only at 0 , then $|\Pi(x)| \leqq|x| \leqq c|\Pi(x)|(x \in X)$ for some $c>0$. Let $P$ denote the complex projective space of hyperplanes of $\mathbb{C}^{n}$ through 0 and, if $H \in P$, let $X(H)$ denote the subspace $\Pi^{-1}(H)$ of $X$ with the canonical complex structure. Let us put

$B:=\{H \in P: \quad X(H)$ is reduced and irreducible at 0$\}$. Since Prof $\mathcal{O} \geqq 2$ by normality (cf. [G-R], III, 1, Satz 11) and since the completion $\hat{\mathcal{O}}$ is also an integral domain by Nagata's theorem (cf. [Ma], 1), we may apply (3.1) to $\mathcal{O}$ and its parameter system induced from the affine coordinates of $\boldsymbol{C}^{n}: B$ includes a nonempty Zariski open subset of $P$. If $H \in B$, there exist $a(H) \geqq 1, b(H) \geqq 0$ such that

$$
a(H)\left(\nu_{X(H)}(u)+\nu_{X(H)}(v)\right)+b(H) \geqq \nu_{X(H)}(u v) \quad\left(u, v \in \mathcal{O}_{X(H), 0}\right)
$$

by the inductive hypothesis. $\left(\nu(), \nu_{H}()\right.$ and $\nu_{X(H)}()$ denote the algebraic orders respectively on $X, H$ and $X(H)$ at 0 .) If we put $B_{h}=\{H: a(H) \leqq h, b(H) \leqq h\}$, we have $B=\underset{1 \leqq h<\infty}{\bigcup} B_{h}$. Therefore there exists $k \in N$ such that $B_{k}$ is an infinite set.

Now suppose that $f, g \in \mathcal{O}$ and $\nu(f g)=p$. Since $\nu_{X(H)}(f g \mid X(H))$ $\geqq \nu(f g)$ by the pullback inequality $\left(\mathrm{I}_{2}\right)$, we have

$$
\mathrm{k}\left(\nu_{X(H)}(f \mid X(H))+\nu_{X(H)}(g \mid X(H))\right)+k \geqq p \quad\left(H \in B_{k}\right) .
$$

Then, we may assume that $k \nu_{X(H)}(f \mid X(H))+k / 2 \geqq p / 2\left(H \in B^{\prime}\right)$ for some infinite subset $B^{\prime} \subset B_{k}$ without loss of generality. Let $s$ denote the order of the covering $X-\Pi^{-1}(D) \rightarrow V-D$ and let $\sigma_{i}(y)(1 \leqq i \leqq s)$ denote the i-th elementary symmetric polynomial of values of $f$ on the fiber $\Pi^{-1}(y)=\left\{x_{1}, \cdots, x_{s}\right\}$ for $y \in V-D . \quad \sigma_{i}(y)$ has a holomorphic 
extension over $V$. If $H \in B^{\prime}$,

$$
\mu_{X(H) \mid, 0}(f \mid X(H)) \geqq \nu_{X(H)}(f \mid X(H)) \geqq(p-k) / 2 k
$$

by $\left(I_{3}\right)$. Hence there exist $\varepsilon_{H}>0$ and $c_{H}>0$ such that

$$
|f(x)| \leqq c_{H}|x|^{(p-k) / 2 k} \quad\left(x \in|X(H)|, \quad|x|<\varepsilon_{H} ; H \in B^{\prime}\right) .
$$

Then there exists $\varepsilon_{H}^{\prime}>0$ such that

$$
\begin{array}{r}
\frac{\left|\sigma_{i}(y)\right|}{(c|y|)^{i(p-k) / 2 k}} \leqq \sum_{\alpha_{1} \leqq \ldots \leqq \alpha_{i}} \frac{\left|f\left(x_{\alpha_{1}}\right)\right|}{\left|x_{\alpha_{1}}\right|^{(p-k) / 2 k}} \cdots \frac{\left|f\left(x_{\alpha_{i}}\right)\right|}{\left|x_{\alpha_{i}}\right|^{(p-k) / 2 k}}=\left(\begin{array}{l}
s \\
i
\end{array}\right) c_{H}^{i} \\
\left(y \in|H| \backslash D, \quad y \mid<\varepsilon_{H}^{\prime} ; H \in B^{\prime}\right) .
\end{array}
$$

Since $\{H:|H| \subset D\}$ is a finite set, by a choice of $B^{\prime}$, we may assume that $D \cap|H|$ is thin in $|H|$ for $H \in B^{\prime}$. Then, if $H \in B^{\prime}$, we have $\left|\sigma_{i}(y)\right| \leqq d_{H, i}|y|^{i(p-k) / 2 k}\left(y \in|H|,|y|<\varepsilon_{H}^{\prime}\right)$ for some $d_{H, i}>0$. This proves that

$$
\nu_{H}\left(\sigma_{i} \mid H\right)=\mu_{|H|, 0}\left(\sigma_{i} \mid H\right) \geqq i(p-k) / 2 k \quad\left(H \in B^{\prime}\right) .
$$

(The left equality follows from the regularity of $H$.) Thus we have $\nu\left(\sigma_{i} \circ \Pi\right) \geqq \nu_{c^{n}}\left(\sigma_{i}\right) \geqq i(p-k) / 2 k$ by $\left(\mathrm{I}_{2}\right)$ and (3.2). Since $f^{s}-\left(\sigma_{1} \circ \Pi\right) f^{s-1}$ $+\cdots \pm \sigma_{s} \circ \Pi=0$ on $X_{0}, \bar{\nu}(f) \geqq(p-k) / 2 k$ by (1.2), (i) $\Rightarrow(r)$. Then we obtain $\left(\mathrm{CI}_{1}\right)$ for the case $n$, using $\left(\mathrm{CI}_{0}\right): \bar{\nu}(f) \leqq \nu(f)+b_{0}$.

(3.5) Corollary. Let $X$ be a reduced complex space and let $E \subset|X|$ be an exceptional set such that the set of regular points of $X$ is connected in an arbitrarily small neighbourhood of $E$. Then for any $\xi, \xi^{\prime} \in E$ there exist $a \geqq 1, b \geqq 0$ such that $\nu_{\xi}(f) \leqq a \nu_{\xi^{\prime}}(f)+b$ for any $f \in \Gamma\left(U, \mathcal{O}_{X}\right)$ defined in a neighbourhood $U$ of $E$.

Proof. By the definition of exceptional set ([G], p. 339), there exists a proper surjective morphism $\Phi: X \rightarrow Y$ such that $\Phi(E)=\{\eta\}$, $\Phi$ is biholomorphic on $|X|-E$ and, if $V$ is a neighbourhood of $\eta$, any $f \in \Gamma\left(\Phi^{-1}(V), \mathcal{O}_{X}\right)$ can be expressed as $f=g \circ \Phi$ for some $g \in$ $\Gamma\left(V, \mathcal{O}_{Y}\right)$. Since $U$ can be expressed as $\Phi^{-1}(V)$, we may apply this to given $f$. Obviously $Y_{\eta}$ is reduced and irreducible and $\operatorname{grnk}_{x} \Phi=$ $\operatorname{dim} Y_{\eta}(x \in E)$. Then $\nu_{\xi}(f)$ and $\nu_{\xi^{\prime}}(f)$ are linearly comparable to $\nu_{\eta}(g)$ by $\left(\mathrm{I}_{2}\right)$ and $\left(\mathrm{CI}_{2}\right)$ of the theorem. Hence $\nu_{\xi}(f)$ and $\nu_{\eta^{\prime}}(f)$ are also linearly comparable.

(3.6) Corollary. Let $A$ be a ring finitely generated over $\boldsymbol{C}$ and $\mathfrak{m}$ one 
of its maximal ideal. Let us put $\nu_{\mathrm{m}}(f)=\sup \left\{p: f \in \mathfrak{m}^{p}\right\}$ for $f \in A$. Then the following conditions are equivalent.

(i) There exist $a_{1} \geqq 1, b_{1} \geqq 0$ such that $\nu_{\mathrm{m}}(f g) \leqq a_{1}\left(\nu_{\mathrm{m}}(f)+\nu_{\mathrm{m}}(g)\right)+b_{1}$ $(f, g \in A)$.

(ii) The completion $\left(A_{\mathrm{m}}\right)^{\wedge}$ of the localization $A_{\mathrm{m}}$ is an integral domain.

Proof. We may assume that $A=\mathbb{C}[x] / I\left(x=\left(x_{1}, \cdots, x_{m}\right)\right)$. Since $\mathbb{C}$ is algebraically closed we may assume that $I \subset \mathfrak{m}_{0}:=\left(x_{1}, \cdots, x_{m}\right) \mathbb{C}[x], \mathfrak{m}=\mathfrak{m}_{0} A$ without loss of generality. Then $\tilde{A}=\mathbb{C}\{x\} / I \mathbb{C}\{x\}$ is an analytic local algebra with the maximal ideal $\mathfrak{m} \tilde{A}$ such that $A_{\mathrm{m}} \subset \tilde{A_{\circ}} \quad\left(A_{\mathrm{m}}\right)^{\wedge}$ is the completion of both $\tilde{A}$ and $A_{\mathrm{m}}$ and hence faithfully flat over them. Then $\tilde{A}$ is faithfully flat over $A_{\mathrm{m}}$ and $\mathfrak{m}^{p} A_{\mathrm{m}}=\mathfrak{m}^{p} \tilde{A} \cap A_{\mathrm{m}}$. On the other hand symbolic powers $\mathfrak{m}^{(p)}:=\mathfrak{m}^{p} A_{\mathfrak{m}} \cap A$ coincide with $\mathfrak{m}^{p}$ by the maximality of $\mathfrak{m}$. These prove that $\nu_{\mathrm{m}}(f)=\nu_{\mathrm{m} A}(f)=\nu_{\mathrm{m}} \tilde{A}(f) \quad(f \in A)$. Then (i) follows from (ii) by $\left(\mathrm{CI}_{1}\right)$ for the integral domain $\tilde{A}$.

Conversely suppose that (ii) is false. Then there exist $\hat{f}, \hat{g} \in\left(A_{\mathrm{m}}\right)^{\wedge}$ such that $\hat{f} \hat{g}=0, \hat{f} \neq 0, \hat{g} \neq 0$. We can identify $\left(A_{\mathrm{m}}\right)^{\wedge}$ with $\mathbb{C}[[x]] /$ $I \mathbb{C}[[x]]$. Take representatives $f, g \in \mathbb{C}[[x]]$ of $\hat{f}, \hat{g}$ respectively such that $\nu_{\mathfrak{m}^{\prime}}(f)=\nu_{\hat{\mathrm{m}}}(\hat{f}), \nu_{\mathrm{m}^{\prime}}(g)=\nu_{\hat{\mathrm{m}}}(\hat{g}) \quad\left(\mathfrak{m}^{\prime}=\mathfrak{m}_{0} \mathbb{C}[[x]], \widehat{\mathfrak{m}}=\mathfrak{m}\left(A_{\mathfrak{m}}\right)^{\wedge}\right)$. Let $f_{p}$ (resp. $g_{p}$ ) be the polynomial which consists of the homogeneous terms of $f$ (resp. $g$ ) of degree less than $\mathrm{p}+1$. If $p \geqq \max \left\{\nu_{\hat{\mathrm{m}}}(\hat{f}), \nu_{\hat{\mathrm{m}}}(\hat{\mathrm{g}})\right\}$, then $\nu_{\mathrm{m}}\left(f_{p} \bmod I\right)=\nu_{\hat{\mu}}(\dot{f})<\infty, \nu_{\mathrm{m}}\left(g_{p} \bmod I\right)=\nu_{\hat{\mathrm{u}}}(\hat{g})<\infty$. On the other hand $\nu_{\mathrm{m}}\left(f_{p} g_{p} \bmod I\right) \geqq p+1$. Hence (i) does not hold.

\section{§4. Real Analytic Algebras}

Let $\mathcal{O}$ be a local real analytic algebra. It can be considered as the local ring $\mathcal{O}_{X, \xi}$ of a germ $X_{\xi}$ of a real analytic space. We define the dimension of $X_{\xi}$ by the Krull dimension of $\mathcal{O}: \operatorname{dim} X_{\xi}:=\operatorname{dim} \mathcal{O}$. In general $\operatorname{dim} X_{\xi} \geqq \operatorname{dim}|X|_{\xi^{\circ}}$ We call $\mathbb{C} \otimes_{\boldsymbol{R}} \mathcal{O}$ the complexification of $\mathcal{O}$. It determines a germ $X_{\xi}^{c}$ of a complex space called the complexification of $X_{\xi}$. Since $\mathbb{C} \otimes_{\boldsymbol{R}} \mathcal{O}$ is integral over $\mathcal{O}$,

$$
\operatorname{dim} X_{\xi}^{c}=\operatorname{dim} \mathbb{C} \otimes_{\boldsymbol{R}} \mathcal{O}=\operatorname{dim} \mathcal{O}=\operatorname{dim} X_{\xi}
$$

by a theorem of Krull-Cohen-Seidenberg.

(4.1) Theorem. Let $\mathcal{O}$ be a local real analytic algebra of positive 
dimension whose complexification is an integral domain. Then $\mathcal{O}$ satisfies the real versions of (1), (2), (3) of the main theorem (3.4).

Proof. (1) Obvious by (3.4), (1).

(2) Let $\Phi^{c}, f^{c}$ be the complexifications of $\Phi$ and $f$ respectively. Since $\nu_{\xi}\left(f^{c}\right)=\nu_{\xi}(f), \nu_{\eta}\left(f^{c} \circ \Phi^{c}\right)=\nu_{\eta}(f \circ \Phi), \operatorname{grnk}_{\eta} \Phi^{c} \geqq \operatorname{grnk}_{\eta} \Phi$ (cf. $\left[\mathrm{I}_{1}\right]$, p. 461 , p. 465 (correction: paracompact $\rightarrow \sigma$-compact)), our assertion follows from (3.4), (2).

(3) This follows from (3.4), (1) and $\left[\mathrm{I}_{1}\right],(3.1)$.

If $S$ is a germ of a real analytic set at $0 \in \boldsymbol{R}^{n}, I(S)$ denotes the ideal of elements of $\mathbb{R}\{x\}$ vanishing on $S$. If $J$ is an ideal of $\boldsymbol{R}\{x\}$, $V(J)$ denotes the maximal germ of a real analytic set at 0 on which all elements of $J$ vanish.

(4.2) Lemma. ([Ri], (4.1)). The following conditions are equivalent for an ideal $J$ of $R\{x\}$.

$$
f_{1}^{2}+\cdots+f_{p}^{2} \in J \text { implies } f_{1}, \cdots, f_{p} \in J \text {. }
$$

$$
J=I(S)
$$

for some germ $S$ of a real analytic set.

$J$ is called real if it satisfies the conditions in (4.2).

(4.3) Lemma. Let $J$ be a prime ideal of $\mathbb{R}\{x\}$.

(i) $J$ is real if and only if $\operatorname{dim} \boldsymbol{R}\{x\} / J=\operatorname{dim} V(J) \quad\left(\left[\mathbf{R}_{i}\right],(4.3)\right)$.

(ii) If $J$ is real, $\boldsymbol{C} \otimes_{\boldsymbol{R}} J$ is prime in $\boldsymbol{C}\{x\}\left([\mathrm{G}-\mathrm{T}]\right.$, p. 371; $\left.\left[\mathrm{R}_{i}\right],(6.1)\right)$.

(4.4) Remark. Let $S$ be an irreducible germ of a real analytic set at $0 \in \mathbb{R}^{n}$. Then (4.1) is applicable to the integral domain $\mathbb{R}\{x\} /$ $I(S)$ by (4.3), (ii). There exists, however, a non-real ideal $J$ with a prime complexification $\boldsymbol{C} \otimes_{\boldsymbol{R}} J$ (e. g. $\left.J=\left(x^{2}+y^{2}+z^{2}\right) \boldsymbol{R}\{x, y, z\}\right)$. In such a case $\operatorname{dim}|X|_{\xi}<\operatorname{dim} X_{\xi}, \operatorname{grnk}_{\eta} \Phi<\operatorname{dim} X_{\xi}$ and hence (2) and (3) of (4.1) is meaningless. Note also that in such a case the complexifications of $X_{\xi}$ and $|X|_{\xi}$ are not compatible.

For $f(z)=\sum \alpha_{k} z^{k} \in \boldsymbol{C}\{z\}\left(z=\left(z_{1}, \cdots, z_{m}\right)\right)$, we put

$$
\begin{aligned}
& f^{*}(z):=\sum \bar{\alpha}_{k} z^{k} \in \mathbb{C}\{z\}, \\
& \operatorname{Re} f(x+i y):=\left(f(x+i y)+f^{*}(x-i y)\right) / 2 \in \boldsymbol{R}\{x, y\},
\end{aligned}
$$




$$
\operatorname{Im} f(x+i y):=\left(f(x+i y)-f^{*}(x-i y)\right) / 2 i \in \mathbb{R}\{x, y\} .
$$

If $\mathrm{I}$ is an ideal of $\boldsymbol{C}\{z\}, I^{r}$ denote the ideal of $\mathbb{R}\{x, y\}$ generated by $\{\operatorname{Re} f(x+i y): f \in I\}$ (or, equivalently, by $\{\operatorname{Im} f(x+i y): f \in I\})$. We put

$$
\begin{aligned}
& \mathcal{O}:=\boldsymbol{C}\{z\} / I, \quad \mathcal{O}^{r}:=\mathbb{R}\{x, y\} / I^{r}, \tilde{I}:=\boldsymbol{C} \otimes_{\boldsymbol{R}} I^{r}, \\
& \tilde{\mathcal{O}}:=\boldsymbol{C}\{x, y\} / \tilde{I}=\boldsymbol{C} \otimes_{\boldsymbol{R}} \mathcal{O}^{r} .
\end{aligned}
$$

Further we put

$$
\begin{aligned}
J:= & \{f(u): f(z) \in I\}, \quad J^{*}:=\left\{f^{*}(v): f(z) \in I\right\}, \\
K:= & (J \otimes 1) \boldsymbol{C}\{u, v\}+\left(1 \otimes J^{*}\right) \mathbb{C}\{u, v\} \\
& \subset \boldsymbol{C}\{u\} \hat{\otimes} \boldsymbol{C}\{v\}=\mathbb{C}\{u, v\}
\end{aligned}
$$

$(\otimes, \hat{\otimes}$ : analytic tensor product, see $[\mathrm{G}-\mathrm{R}])$. Let us define an isomorphism $\theta: \mathbb{C}\{u, v\} \rightarrow \mathbb{C}\{x, y\}$ by $\theta(f(u, v)):=f(x+i y, x-i y)$.

(4.5) Lemma. ( $f f .[\mathrm{Ma}], \S 3$, which treats analytic sets)。

$$
\tilde{I}=\theta(K) \quad \text { i.e. } \tilde{\mathcal{O}} \cong \mathcal{O} \otimes \mathcal{O}^{*} \quad\left(\mathcal{O}^{*}=\mathbb{C}\{v\} / J^{*}\right) \text {. }
$$

Proof. Obvious by the following implication.

$$
\begin{aligned}
f \in \theta(K) \Leftrightarrow & \exists g \in K \text { s. t. } f(x, y)=g(x+i y, x-i y) \\
\Leftrightarrow & \exists \alpha_{\mu}, \exists \beta_{\mu} \in C\{u, v\} ; \exists h_{\mu}, \exists k_{\mu} \in J(\mu=1, \cdots, p) \\
& \text { s. t. } f(x, y)=\sum_{\mu}\left\{\alpha_{\mu}(x+i y, x-i y) h_{\mu}(x+i y)\right. \\
& \left.\quad+\beta_{\nu}(x+i y, x-i y) k_{\mu}^{*}(x-i y)\right\} \\
\Leftrightarrow & f \in \tilde{I} .
\end{aligned}
$$

(4.6) Proposition. If $\mathcal{O}$ is an integral domain in the above, we have the following.

(i) $\tilde{\mathcal{O}}$ is an integral domain.

(ii) $I^{r}$ is a real ideal.

(ii) is just the affirmative answer to Malgrange's conjecture [Ma], p. 124.

Proof. (i) By (4.5) we have only to prove that $\mathcal{O} \hat{\otimes} \mathcal{O}^{*}$ is an integral domain. Since $\mathcal{O}^{*}$ is isomorphic to $\mathcal{O}$ as a ring, it is an integral domain. Then $\mathcal{O} \hat{\otimes} \mathcal{O}^{*}$ is also so by Nagata's theorem $\left[\mathrm{N}_{2}\right]$, (47. 5). 
(ii) Since $\tilde{\mathcal{O}}$ is an integral domain and since $\operatorname{dim} \mathcal{O}^{r}=\operatorname{dim} \tilde{\mathcal{O}}=\operatorname{dim} \mathcal{O} \hat{\otimes} \mathcal{O}^{*}=2 \operatorname{dim} \mathcal{O}=\operatorname{dim} V\left(I^{r}\right)$, we have the assertion by (4.3), (i).

(4.7) Remark. If $\mathcal{O}$ is an integral domain, we may well call $\mathcal{O}^{r}$ the real analytic algebra associated to $\mathcal{O}$. But, if not, $\mathcal{O}^{r}$ is not fit to be called so. For example, consider the case $I=I_{1} \cap I_{2}, I_{i}=z_{i} \mathbb{C}$ $\left\{z_{1}, z_{2}\right\}(i=1,2)$. Since $K=\left(u_{1} u_{2}, v_{1} v_{2}\right) \mathbb{C}\{u, v\}, V(\tilde{I}) \cong V(K)$ has four components at $0 \in \mathbb{C}^{4}$. Therefore $V(\tilde{I})$ is bigger than the join of $V\left(\tilde{I}_{1}\right)$ and $V\left(\tilde{I}_{2}\right)$. Returning to the algebras of the real parts, we see that $\mathcal{O}^{r}$ is too big to be called the real analytic space associated to $\mathcal{O}$.

(4.8) Remark. If $f_{\mu}(\mu=1, \cdots, p)$ generate $I, \operatorname{Re} f_{\mu}(x+i y), \operatorname{Im} f_{\mu}$ $(x+i y)$ generate $I^{r}$. Then, for any complex space $X$, we can construct the real analytic space $X_{r}$ (defined by a coherent sheaf of ideals) and its complexification $\tilde{X}$ in the sense of $[\mathrm{H}]$.

Proof of (3.4), (3). Let $X_{\xi}$ be a reduced and irreducible germ of a complex space. Then $X_{\xi}^{r}$ has a reduced and irreducible complexification $\tilde{X}_{\xi}$ by $(4.6)$. Hence (4.1) holds for $X_{\xi \text {. }}^{r}$ If $f \in \mathcal{O}_{X, \xi}$, we have only to apply $(4.1)$, (3) for $X_{\xi}^{r}$ to the real and the imaginary part of $f$. (If we consider the proof of (4.1), (3), we see that (3.4), (3) for $n$-dimensional $X_{\xi}$ results from $(3.4)$, (2) for $2 n$-dimensional $\tilde{X}_{\xi}$.)

\section{\$5. Nearmess to $\mathbb{p}^{-\mathrm{th}}$ Power in $\mathbb{C}\left\{x_{1}, \cdots, x_{m}\right\}$}

In this section we put $A=\mathcal{O}_{m, 0}=\mathbb{C}\left\{x_{1}, \cdots, x_{m}\right\}, \hat{A}=\mathbb{C}\left[\left[x_{1}, \cdots, x_{m}\right]\right]$, $\nu(\quad)=\nu_{0}()$. Let $p$ be a prime number and $u$ not a $p$-th power in $A$. The functional equation $f^{p} u-g^{p}=0$ has unique solution $(f, g)$ $=(0,0)$. If $\nu\left(f^{p} u-g^{p}\right)$ is large, $(f, g)$ is a "near solution". We claim that a near solution is near to the unique solution:

(5.1) Proposition. If $u \in A$ is not a $p$-th power in $A$, there exist

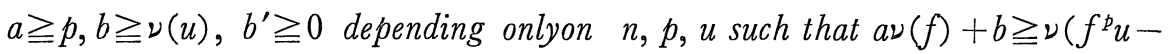
$\left.g^{p}\right), a \nu(g)+b^{\prime} \geqq \nu\left(f^{p} u-g^{p}\right)$ for any $f, g \in \hat{A}$.

(5.2) Remark. By Artin's strong approximation theorom [ $\left.\mathrm{A}_{2}\right]$, 
(6. 1), we see that, if $u$ is a polynomial, there exists a function $e$ : $N \rightarrow \mathbb{N}$ such that $\nu(f), \nu(g) \geqq e\left[\nu\left(f^{p} u-g^{p}\right)\right]$. (5.1) asserts that we can choose a linear $e$.

Proof. Let us put $F=\nu(f), G=\nu(g), K=\nu\left(f^{p} u-g^{p}\right), U=\nu(u)$ and $T=\max \{F, G, K\}+1$. We have to prove that

$$
a F+b \geqq K, \quad a G+b^{\prime} \geqq K .
$$

Considering the cases $g=0$ and $f=0$ we have necessary conditions

$$
a \geqq p, b \geqq \nu(u), b^{\prime} \geqq 0 .
$$

This assures $\left(^{*}\right)$ if $T=\infty$. Thus we have only to treat the case $T<\infty$. If $f-f^{\prime}, g-g^{\prime} \in \mathfrak{m}^{T}$ then $\nu\left(f^{\prime}\right)=F, \nu\left(g^{\prime}\right)=G, \nu\left(f^{\prime p} u-g^{\prime p}\right)=K_{0}$ : Hence we may assume that $f, g \in A$. If the initial form (the homogeneous term of the lowest degree) In $u$ is not a $p$-th power, $K=\min$ $(p F+U, p G)$ and $\left(^{*}\right)$ is obvious $(a=p)$. So we may assume that $U=p V(V \in \mathbb{N})$.

Let us introduce a new variable $z$ and consider

$$
\begin{aligned}
& \mathcal{O}_{m+1,0}:=\boldsymbol{C}\left\{x_{1}, \cdots, x_{m}, z\right\}, \\
& \mathcal{O}:=\mathcal{O}_{m+1,0} /\left(u-(-z)^{p}\right) \mathcal{O}_{m+1,0} .
\end{aligned}
$$

Let $\nu$ and $\nu_{\mathcal{O}}$ denote the algebraic order of $\mathcal{O}_{m+1,0}$ and $\mathcal{O}$ with respect to the respective maximal ideals (these are extensions of the original ע). $h \in \mathcal{O}$ has a unique representative of form

$$
\sum_{i=0}^{p-1} h_{i} z^{i} \in \mathcal{O}_{m+1,0} \quad\left(h_{i} \in A\right) .
$$

$\nu_{\mathcal{O}}(h)=\min \left(\nu\left(h_{1}\right)+i\right)$ by the assumption $U=p V \geqq p$. If we put

$$
\begin{aligned}
h:= & \left(f g^{p-2} z^{p-1}-g^{p-1} z^{p-2}\right)+\left(f^{p-1} u z^{p-3}-f^{p-2} g u z^{p-4}\right. \\
& \left.\left.+\cdots+f^{3}(-g)^{p-4} u z+f^{2}(-g)^{p-3} u\right) \quad \text { (if } p=2, h:=f z-g\right),
\end{aligned}
$$

we have

$$
(f z+g) h \equiv\left(f^{p} u-g^{p}\right) z^{p-2} \bmod u-(-z)^{p} \quad(f, g \in A) .
$$

Then, applying $(3.4),(1)$ to the integral domain $\mathcal{O}$, we have

$$
\begin{array}{r}
a_{1}(\nu(f z+g)+\nu(h))+b_{1} \geqq \nu\left(\left(f^{p} u-g^{p}\right) z^{p-2}\right)=K+p-2 \\
(f, g \in A) .
\end{array}
$$

If $p G \neq p F+U$, we can directly prove (*) using (i). So we have only to treat the case $p G=p F+U$. Then, since 


$$
\begin{aligned}
& \nu\left(g^{p-1} z^{p-2}\right)-\nu\left(f g^{p-2} z^{p-1}\right)=V-1, \\
& \nu\left(f^{p-1} u z^{p-3}\right)-\nu\left(f g^{p-2} z^{p-1}\right)=2(V-1) \\
& \nu\left(f^{p-2} g u z^{p-4}\right)-\nu\left(f g^{p-2} z^{p-1}\right)=3(V-1), \\
& \quad \cdots\left(f^{2} g^{p-3} u\right)-\nu\left(f g^{p-2} z^{p-1}\right)=(p-1)(V-1),
\end{aligned}
$$

we have

(iii)

$$
\begin{aligned}
\nu(h) & =\nu\left(f g^{p-2} z^{p-1}\right)=(p-1) F+(p-2) V+p-1 \\
& =(p-1) G-V+p-1 .
\end{aligned}
$$

Since $G-(F+1)=V-1 \geqq 0$,

$$
\nu(f z+g)=F+1=G-V+1 .
$$

Combining (ii), (iii) and (iv), we have

$$
\begin{aligned}
& a_{1}(p F+(p-2) V+p)+b_{1}-p+2 \geqq K, \\
& a_{1}(p G-2 V+p)+b_{1}-p+2 \geqq K .
\end{aligned}
$$

Hence $\left(^{*}\right)$ holds by a suitable choice of $a$ and $b \quad\left(a=a_{1} p\right)$.

(5.3) Definition. $\theta(p, u):=\inf a / p(a:$ in $(5.1))$.

This can be regarded as a measure of nearness to $p$-th power. In view of the proof above, we have an estimate $1 \leqq \theta(p, u) \leqq a_{1}$.

(5.4) Example. If In $u$ is not a $p$-th power, $\theta(p, u)=1$. This means that $u$ is very far from $p$-th powers.

(5.5) Example. Suppose that $u:=v^{p}-w$ is not a $p$-th power in $A$ and $k:=\nu(w) \geqq p \nu(v)+1$. Using Tailor expansion

$$
p \sqrt{1+t+t^{2}+\cdots}=1+c_{1} t+c_{2} \mathrm{t}^{2}+\cdots
$$

we have

$$
\begin{aligned}
& \left(1+c_{1} t+c_{2} t^{2}+\cdots+c_{n} t^{n}\right)^{p}(1-t)-1 \\
& \quad=d_{n+1} t^{n+1}+d_{n+2} t^{n+2}+\cdots+d_{n p+1} t^{n p+1}
\end{aligned}
$$

for suitable $d_{n+1}, \cdots, d_{n p+1} \in \boldsymbol{C}$. Hence

$$
\begin{aligned}
& \left(v^{n p}+c_{1} v^{(n-1) p} w+c_{2} v^{(n-2) p} w^{2}+\cdots+c_{n} w^{n}\right)^{p}\left(v^{p}-w\right)-v^{(n p+1) p} \\
& =d_{n+1} v^{(n p-n) p} w^{n+1}+d_{n+2} v^{(n p-n-1) p} w^{n+2}+\cdots+d_{n p+1} w^{n p+1} .
\end{aligned}
$$

If we put $f_{n}=v^{n p}+c_{1} v^{(n-1) p} w+\cdots+c_{n} w^{n}, g_{n}=v^{n p+1}$, we have 


$$
\nu\left(f_{n}^{p} u-g_{n}^{p}\right)=\nu\left(v^{(n p-n) p} w^{n+1}\right)=n(p-1) p h+(n+1) k \quad(h:=\nu(\nu)) .
$$

Hence $\theta(p, u) \geqq\left(p^{2} h-p h+k\right) / p^{2} h=1+(k-h p) / h p^{2}(>1)$.

(5.6) Example. If $u=x_{1}^{p}-x_{2}^{k}(k>p, k \neq 0 \bmod p), a_{1}$ for $\mathcal{O}$ in the proof of $(5.1)$ is estimated as $a_{1} \leqq k / p$ by the proof of $\left[\mathrm{I}_{1}\right],(1.5)$. Hence we have

$$
1-1 / p+k / p^{2} \leqq \theta(p, u) \leqq k / p 。
$$

\section{Appendix : inf $a_{1}$ and inf $b_{1}$ for Some Elementary Singularities}

Let $a_{1}$ denote the infimum of $a_{1}$ in (3.4) for a given local complex analytic integral domain $\mathcal{O}=\mathcal{O}_{X, \xi}$. In case $\underline{a}_{1}$ is attained, $\underline{b}_{1}$ denotes the infimum of $b_{1}$ that satisfies (3.4), (1) with $a_{1}=a_{1}$. The author does not know whether $\underline{a}_{1}$ is always attained and whether $\underline{a}_{1}$ is a rational number. Here we exhibit the explicit values of $a_{1}$ and $\underline{b}_{1}$ for some elementary singularities. $a_{1}$ in (i), (iii), (iv) and $\underline{b}_{1}$ in (i) were first taught by Tomari.

(i) $\nu(f g)=\nu(f)+\nu(g) \quad(f, g \in \mathcal{O})$ (i. e. $a_{1}$ and $\underline{b}_{1}$ are attained and $\left.\underline{a}_{1}=1, \underline{b}_{1}=0\right)$ iff $\operatorname{Gr}_{m} \mathcal{O}:=\sum_{p=0}^{\infty} \mathfrak{m}^{p} / \mathfrak{m}^{p+1}$ is an integral domain. The proof is standard.

(ii) If $\operatorname{dim} \mathcal{O}=1$, the exceptional fiber of the normalization of the blowing-up of $X$ with center $\mathfrak{m}_{\xi}$ is irreducible. Then $a_{1}$ is attained and $\underline{a}_{1}=1$ by $(1.4)$.

(iii) Let $(X, 0)$ be a hypersurface singularity in $\mathbb{C}^{3}$ defined by $z^{2}-\varphi(x, y)=0(\varphi(0,0)=0)$. Let $\nu$ and $\nu_{\mathcal{O}}$ denote the algebraic orders on $\mathbb{C}\{x, y, z\}$ and on $\mathcal{O}:=\mathbb{C}\{x, y, z\} /\left(z^{2}-\varphi\right) \mathbb{C}\{x, y, z\}$ respectively. Further we assume that $r:=\nu(\varphi)$ is an odd number greater than 2. Here we claim about $\mathcal{O}$ that $\underline{a}_{1}=1$ and $\underline{b}_{1}=r-2$ and that they are attained. Obviously every $\bar{f} \in \mathcal{O}$ has a unique representative of the form $f=f_{1}(x, y) z+f_{2}(x, y) \in \mathbb{C}\{x, y, z\}$ such that $\nu_{\mathcal{O}}(\bar{f})=\nu(f)=\min \left(\nu\left(f_{1}\right)\right.$ $\left.+1, \nu\left(f_{2}\right)\right)$. Suppose that

$$
\nu_{\mathcal{O}}(\bar{f} \bar{g})>\nu_{\mathcal{O}}(\bar{f})+\nu_{\mathcal{O}}(\bar{g})+r-2
$$

for some $\bar{f}, \bar{g} \in \mathcal{O}$. This implies that

$$
\begin{aligned}
& \nu\left(f_{1} g_{2}+f_{2} g_{1}\right)>\nu(f)+\nu(g)+r-3, \\
& \nu\left(f_{1} g_{1} \varphi+f_{2} g_{2}\right)>\nu(f)+\nu(g)+r-2 .
\end{aligned}
$$


By a standard calculation we have

$$
\nu\left(f_{1}^{2} \varphi-f_{2}^{2}\right)>2 \nu(f)+r-2 .
$$

On the other hand, since $r$ is odd,

$$
\nu\left(f_{1}^{2} \varphi-f_{2}^{2}\right)=\min \left(2 \nu\left(f_{1}\right)+r, 2 \nu\left(f_{2}\right) \leqq 2 \nu(f)+r-2,\right.
$$

a contradction. Thus (*) never holds and we may put $a_{1}=1, b_{1}=r-2$. Since

$$
\nu_{\mathcal{O}}\left(z^{2}\right)=r=\left(\nu_{\mathcal{O}}(z)+\nu_{\mathcal{O}}(z)\right)+r-2,
$$

$b_{1}$ can never be smaller than $r-2$. Hence we have proved the assertion.

In the case 0 is an isolated singularity of $X$ (i. e. $X$ is normal at 0 ), we can prove that the exceptional fiber of the normalization of the blowing-up of $X$ with center $\mathfrak{m}_{0}$ is irreducible, which again implies that $\underline{a}_{1}$ is attained and $\underline{a}_{1}=1$. This case includes the rational singularities of types $D_{k}(k \geqq 4), E_{6}, E_{7}, E_{8}$ (cf. [Br]).

(iv) The remaining two dimensional rational singularity of multiplicity 2 is $A_{k}(k \geqq 1)$ defined by the equation $x^{2}+y^{2}+z^{k+1}=0$ in $\mathbb{C}^{3}$. By the proof of $\left[\mathrm{I}_{1}\right]$, (1.5), we see that $(3.4)$, (1) holds for $a_{1}$ $=(k+1) / 2, b_{1}=0$. On the other hand

$$
\begin{aligned}
a_{1} & \geqq\left\{\nu\left((x+i y)^{p}(x-i y)^{p}\right)-b_{1}\right\} /\left\{\nu\left((x+i y)^{p}\right)+\nu\left((x-i y)^{p}\right)\right\} \\
& =\left\{(k+1) p-b_{1}\right\} / 2 p \quad(p \in \mathbb{N}) .
\end{aligned}
$$

Hence $a_{1} \geqq(k+1) / 2$. Thus we have proved $\underline{a}_{1}=(k+1) / 2, \underline{b}_{1}=0$ and they are attained.

\section{References}

[A] Artin, M., Algebraic approximation of structures over complete local rings, Publ. Math. IHES, 36 (1969), 23-58.

[Be] Becker, J., On the composition of power series, In : Commutative algebra (analytic methods) (LN in pure and ap. math. 68), (159-172), Marcel Dekker New York, 1982.

[Br] Brieskorn, E., Über die Auflösung gewisser Singularitäten von holomorphen Abbildungen, Math. Ann., 166 (1966), 76-102.

[F] Flenner, H., Die Sätze von Bertini für lokale Ringe, Math. Ann., 229 (1977), 97-111.

[G] Grauert, H., Über Modifikationen und exceptionelle analytische Mengen, Math. Ann., 146 (1962), 331-368.

[G-R] Grauert, H. and Remmert, R., Analytische Stellenalgebren (GMW 176), Springer, Berlin-Heidelberg-New York, 1971.

[G-T] Galbiati, M., Tognoli, A., Alcune proprietà delle varietà algebriche reali, Ann. Scuola Norm. Sup. Pisa, 27 (1973), 359-404.

[H] Hironaka, H., Subanalytic sets, In: Number theory, algebraic geometry and commutative algebra, in honor of Y. Akizuki, (453-493), Kinokuniya, Tokyo, 1973. 
$\left[\mathrm{I}_{1}\right]$ Izumi, S., Linear complementary inequalities for orders of germs of analytic functions, Invent. Math., 65 (1982), 459-471.

$\left[\mathrm{I}_{2}\right] \longrightarrow$ Inequalities for orders on a rational singularity of a surface, J. Math. Kyoto Univ., 24 (1984), 239-241.

[L-T] Lejeune-Jalabert, M. and Teissier, B., Clôture intégrale des idéaux et équisingularite, École Polytechnique 1974.

[Ma] Malgrange, B., Sur les fonctions différentiables et les ensenbles analytiques, Bull. Soc. Math. France, 91 (1963), 113-127.

[Mu] Mumford, D., The topology of normal singularities of an algebraic surface and a criterion for simplicity, Pub. Math. IHES, 11 (1961), 229-246.

[N $\left.\mathrm{N}_{1}\right]$ Nagata, M., Note on a paper of Samuel concerning asymptotic properties of ideals, Mem. Coll. Sci. Univ. Kyoto, Ser. A, 30 (1957), 165-175.

$\left[\mathrm{N}_{2}\right]$, Local rings (Interscience tracts in Pure \& applied Math. 13), Interscience Publishers, New York-London, 1962.

[Re $\left.\mathrm{R}_{1}\right]$ Rees, D., Valuations associated with a local ring (I), Proc. London Math. Soc., 5(1955), 107-128.

[Re $\left.\mathrm{Re}_{2}\right]$, Valuations associated with ideals (II), J. London Math. Soc., 31 (1956), 221-228.

$\left[\mathrm{Re}_{3}\right]$ - Valuations associated with a local ring (II), J. London Math. Soc., 31 (1956), 228-235.

[Ri] Risler, J-J., Le théorèm des zéros en géométries algébraique et analytique réelles, Bull. Soc. Math. France, 104 (1976), 113-127.

[S] Samuel, P., Some asymptotic properties of powers of ideals, Ann. of Math., 56 (1952), 11-21. 
